\title{
Fractional $q$-difference hybrid equations and inclusions with Dirichlet boundary conditions
}

\author{
Bashir Ahmad ${ }^{1 *}$ and Sotiris K Ntouyas ${ }^{2}$
}

\section{"Correspondence:}

bashirahmad_qau@yahoo.com 'Department of Mathematics,

Faculty of Science, King Abdulaziz University, P.O. Box 80203, Jeddah, 21589, Saudi Arabia

Full list of author information is available at the end of the article

\begin{abstract}
We study the existence of solutions for boundary value problems of nonlinear fractional q-difference hybrid equations and inclusions by means of fixed point theorems for single- and multi-valued maps. The main results are illustrated with the aid of examples.

MSC: $34 \mathrm{~A} 60 ; 34 \mathrm{~A} 08 ; 34 \mathrm{~B} 18$

Keywords: fractional derivative; q-difference; boundary conditions; fixed point; existence
\end{abstract}


Next, we study a boundary value problem of fractional $q$-difference hybrid inclusions given by

$$
\left\{\begin{array}{l}
D_{q}^{\alpha}\left(\frac{x(t)}{f(t, x(t))}\right) \in F(t, x(t)), \quad 0<t<1,1<\alpha \leq 2,0<q<1, \\
x(0)=x(1)=0
\end{array}\right.
$$

where $F:[0,1] \times \mathbb{R} \rightarrow \mathcal{P}(\mathbb{R})$ is a multi-valued map, $\mathcal{P}(\mathbb{R})$ is the family of all nonempty subsets of $\mathbb{R}$.

The paper is organized as follows: in Section 2 we recall some preliminary facts. The existence of solutions for the problem (1.1) is shown in Section 3 while the multi-valued problem (1.2) is investigated in Section 4. The main tool of our study are fixed point theorems due to Dhage for single-valued [27] and multi-valued [28] maps.

\section{Preliminaries}

First of all, we recall the notations and terminology for $q$-fractional calculus [22, 29].

For a real parameter $q \in \mathbb{R}^{+} \backslash\{1\}$, a $q$-real number denoted by $[a]_{q}$ is defined by

$$
[a]_{q}=\frac{1-q^{a}}{1-q}, \quad a \in \mathbb{R}
$$

The $q$-analog of the Pochhammer symbol ( $q$-shifted factorial) is defined as

$$
(a ; q)_{0}=1, \quad(a ; q)_{k}=\prod_{i=0}^{k-1}\left(1-a q^{i}\right), \quad k \in \mathbb{N} \cup\{\infty\} .
$$

The $q$-analog of the exponent $(x-y)^{k}$ is

$$
(x-y)^{(0)}=1, \quad(x-y)^{(k)}=\prod_{j=0}^{k-1}\left(x-y q^{j}\right), \quad k \in \mathbb{N}, x, y \in \mathbb{R} .
$$

The $q$-gamma function $\Gamma_{q}(y)$ is defined as

$$
\Gamma_{q}(y)=\frac{(1-q)^{(y-1)}}{(1-q)^{y-1}}
$$

where $y \in \mathbb{R} \backslash\{0,-1,-2, \ldots\}$. Observe that $\Gamma_{q}(y+1)=[y]_{q} \Gamma_{q}(y)$.

Definition 2.1 ([22]) Let $f$ be a function defined on $[0,1]$. The fractional $q$-integral of the Riemann-Liouville type of order $\beta \geq 0$ is $\left(I_{q}^{0} f\right)(t)=f(t)$ and

$$
I_{q}^{\beta} f(t):=\int_{0}^{t} \frac{(t-q s)^{(\beta-1)}}{\Gamma_{q}(\beta)} f(s) d_{q} s=t^{\beta}(1-q)^{\beta} \sum_{k=0}^{\infty} q^{k} \frac{\left(q^{\beta} ; q\right)_{n}}{(q ; q)_{n}} f\left(t q^{k}\right), \quad \beta>0, t \in[0,1] .
$$

Observe that $\beta=1$ in Definition 2.1 yields the $q$-integral

$$
I_{q} f(t):=\int_{0}^{t} f(s) d_{q} s=t(1-q) \sum_{k=0}^{\infty} q^{k} f\left(t q^{k}\right) .
$$


For more details on $q$-integral and fractional $q$-integral, see Section 1.3 and Section 4.2, respectively, in [22].

Remark 2.2 The $q$-fractional integration possesses the semigroup property ([22], Proposition 4.3):

$$
I_{q}^{\gamma} I_{q}^{\beta} f(t)=I_{q}^{\beta+\gamma} f(t) ; \quad \gamma, \beta \in \mathbb{R}^{+} .
$$

Further, it has been shown in Lemma 6 of [29] that

$$
I_{q}^{\beta}(x)^{(\sigma)}=\frac{\Gamma_{q}(\sigma+1)}{\Gamma_{q}(\beta+\sigma+1)}(x)^{(\beta+\sigma)}, \quad 0<x<a, \beta \in \mathbb{R}^{+}, \sigma \in(-1, \infty) .
$$

Before giving the definition of fractional $q$-derivative, we recall the concept of $q$-derivative.

The $q$-derivative of a function $f(t)$ is defined as

$$
\left(D_{q} f\right)(t)=\frac{f(t)-f(q t)}{t-q t}, \quad t \neq 0, \quad\left(D_{q} f\right)(0)=\lim _{t \rightarrow 0}\left(D_{q} f\right)(t) .
$$

Furthermore,

$$
D_{q}^{0} f=f, \quad D_{q}^{n} f=D_{q}\left(D_{q}^{n-1} f\right), \quad n=1,2,3, \ldots
$$

Definition 2.3 ([22]) The Caputo fractional $q$-derivative of order $\beta>0$ is defined by

$$
{ }^{c} D_{q}^{\beta} f(t)=I_{q}^{\lceil\beta\rceil-\beta} D_{q}^{\lceil\beta\rceil} f(t),
$$

where $\lceil\beta\rceil$ is the smallest integer greater than or equal to $\beta$.

Next we recall some properties involving Riemann-Liouville $q$-fractional integral and Caputo fractional $q$-derivative ([22], Theorem 5.2).

$$
\begin{aligned}
& I_{q}^{\beta c} D_{q}^{\beta} f(t)=f(t)-\sum_{k=0}^{\lceil\beta\rceil-1} \frac{t^{k}}{\Gamma_{q}(k+1)}\left(D_{q}^{k} f\right)\left(0^{+}\right), \quad \forall t \in(0, a], \beta>0 ; \\
& { }^{c} D_{q}^{\beta} I_{q}^{\beta} f(t)=f(t), \quad \forall t \in(0, a], \beta>0 .
\end{aligned}
$$

\section{An existence result for the single-valued problem}

This section begins with a basic result, which plays a pivotal role in the forthcoming analysis. Let $C([0,1], \mathbb{R})$ denote a Banach space of continuous functions from $[0,1]$ into $\mathbb{R}$ with the norm $\|x\|=\sup _{t \in[0,1]}|x(t)|$.

Lemma 3.1 For $y \in C([0,1], \mathbb{R})$, the unique solution of the problem

$$
\left\{\begin{array}{l}
D_{q}^{\alpha}\left(\frac{x(t)}{f(t, x(t))}\right)=y(t), \quad 0<t<1, \\
x(0)=x(1)=0,
\end{array}\right.
$$


is given by

$$
x(t)=f(t, x(t))\left(\int_{0}^{t} \frac{(t-q s)^{(\alpha-1)}}{\Gamma_{q}(\alpha)} y(s) d_{q} s-t \int_{0}^{1} \frac{(1-q s)^{(\alpha-1)}}{\Gamma_{q}(\alpha)} y(s) d_{q} s\right), \quad t \in[0,1] .
$$

Proof It is well known that the general solution of the $q$-fractional differential equation in (3.1) can be written as

$$
x(t)=f(t, x(t))\left(\int_{0}^{t} \frac{(t-q s)^{(\alpha-1)}}{\Gamma_{q}(\alpha)} y(s) d_{q} s+c_{0} t+c_{1}\right), \quad t \in[0,1],
$$

where $c_{0}, c_{1} \in \mathbb{R}$ are arbitrary unknown constants. Using the boundary conditions given in (3.2), we have

$$
c_{1}=0, \quad c_{0}=-\int_{0}^{1} \frac{(1-q s)^{(\alpha-1)}}{\Gamma_{q}(\alpha)} y(s) d_{q} s .
$$

Substituting the values of $c_{0}$ and $c_{1}$ in (3.2), we get

$$
x(t)=f(t, x(t))\left(\int_{0}^{t} \frac{(t-q s)^{(\alpha-1)}}{\Gamma_{q}(\alpha)} y(s) d_{q} s-t \int_{0}^{1} \frac{(1-q s)^{(\alpha-1)}}{\Gamma_{q}(\alpha)} y(s) d_{q} s\right), \quad t \in[0,1] .
$$

We use the nonlinear alternative of Schaefer's type due to Dhage [27].

Lemma 3.2 ([27]) Let $B_{r}(0)$ and $\bar{B}_{r}(0)$ respectively denote an open and closed ball in a Banach algebra $X$ centered at origin of radius $r$, for some real number $r>0$. Let $A: X \rightarrow X$ and $B: \bar{B}_{r}(0) \rightarrow X$ be two operators such that:

(a) A is Lipschitzian with a Lipschitz constant $k$,

(b) B is completely continuous,

(c) $k M<1$, where $M=\left\|B\left(\bar{B}_{r}(0)\right)\right\|=\sup \left\{\|B x\|: x \in \bar{B}_{r}(0)\right\}$.

Then either

(i) the equation $A x B x=x$ has a solution in $\bar{B}_{r}(0)$, or

(ii) there exists an $u \in X$ with $\|u\|=r$ such that $\lambda A u B u=u$ for some $0<\lambda<1$.

Now we are in a position to present the first main result of our paper.

Theorem 3.3 Assume that:

$\left(\mathrm{H}_{1}\right)$ the function $f:[0,1] \times \mathbb{R} \rightarrow \mathbb{R} \backslash\{0\}$ is continuous and there exists a bounded function $\phi$, with bound $\|\phi\|$, such that $\phi(t)>0$ for $t \in[0,1]$ and

$$
|f(t, x)-f(t, y)| \leq \phi(t)|x(t)-y(t)| \text { for } t \in[0,1] \text { and for all } x, y \in \mathbb{R}
$$

$\left(\mathrm{H}_{2}\right)$ there exist a continuous nondecreasing function $\Omega:[0, \infty) \rightarrow(0, \infty)$ and a function $\gamma \in C\left([0,1], \mathbb{R}^{+}\right)$such that

$$
|g(t, x)| \leq \gamma(t) \Omega(\|x\|), \quad t \in[0,1] \text { and for all } x \in \mathbb{R}
$$

$\left(\mathrm{H}_{3}\right)$ there exists a real number $r>0$ such that

$$
r>\frac{\frac{2 F_{0}}{\Gamma_{q}(\alpha+1)}\|\gamma\| \Omega(r)}{1-\frac{2\|\phi\|}{\Gamma_{q}(\alpha+1)}\|\gamma\| \Omega(r)},
$$


where

$$
\frac{2\|\phi\|}{\Gamma_{q}(\alpha+1)}\|\gamma\| \Omega(r)<1, \quad F_{0}=\sup _{t \in[0,1]}|f(t, 0)| .
$$

Then the problem (1.1) has at least one solution on $[0,1]$.

Proof Let us set $X=C([0,1], \mathbb{R})$ and consider a closed ball $\bar{B}_{r}(0)$ in $X$, where $r$ satisfies the inequality (3.3). By Lemma 3.1, the problem (1.1) is equivalent to the integral equation

$$
\begin{aligned}
x(t)= & f(t, x(t))\left(\int_{0}^{t} \frac{(t-q s)^{(\alpha-1)}}{\Gamma_{q}(\alpha)} g(s, x(s)) d_{q} s\right. \\
& \left.-t \int_{0}^{1} \frac{(1-q s)^{(\alpha-1)}}{\Gamma_{q}(\alpha)} g(s, x(s)) d_{q} s\right), \quad t \in[0,1] .
\end{aligned}
$$

Define two operators $\mathcal{A}, \mathcal{B}: \bar{B}_{r}(0) \rightarrow X$ by

$$
\mathcal{A} x(t)=f(t, x(t)), \quad t \in[0,1]
$$

and

$$
\mathcal{B} x(t)=\int_{0}^{t} \frac{(t-q s)^{(\alpha-1)}}{\Gamma_{q}(\alpha)} g(s, x(s)) d_{q} s-t \int_{0}^{1} \frac{(1-q s)^{(\alpha-1)}}{\Gamma_{q}(\alpha)} g(s, x(s)) d_{q} s .
$$

Observe that $x=\mathcal{A} x \mathcal{B} x$. We shall show that the operators $\mathcal{A}$ and $\mathcal{B}$ satisfy all the conditions of Lemma 3.2. The proof is constructed in several steps.

Step 1. $\mathcal{A}$ is Lipschitz on X, that is, the assumption (a) of Lemma 3.2 holds.

Let $x, y \in X$. Then by $\left(\mathrm{H}_{1}\right)$, we have

$$
\begin{aligned}
|\mathcal{A} x(t)-\mathcal{A} y(t)| & =|f(t, x(t))-f(t, y(t))| \\
& \leq \phi(t)|x(t)-y(t)| \\
& \leq\|\phi\|\|x-y\|
\end{aligned}
$$

for all $t \in[0,1]$. Taking the supremum over the interval $[0,1]$, we get

$$
\|\mathcal{A} x-\mathcal{A} y\| \leq\|\phi\|\|x-y\|
$$

for all $x, y \in \bar{B}_{r}(0)$. So $\mathcal{A}$ is Lipschitz on $\bar{B}_{r}(0)$ with Lipschitz constant $\|\phi\|$.

Step 2. The operator $\mathcal{B}$ is completely continuous on $\bar{B}_{r}(0)$, that is, (b) of Lemma 3.2 holds. First we show that $\mathcal{B}$ is continuous on $\bar{B}_{r}(0)$. Let $\left\{x_{n}\right\}$ be a sequence in $\bar{B}_{r}(0)$ converging to a point $x \in \bar{B}_{r}(0)$. Then, by Lebesgue's dominated convergence theorem, we have

$$
\begin{aligned}
& \lim _{n \rightarrow \infty} \mathcal{B} x_{n}(t) \\
& \quad=\lim _{n \rightarrow \infty} \int_{0}^{t} \frac{(t-q s)^{(\alpha-1)}}{\Gamma_{q}(\alpha)} g\left(s, x_{n}(s)\right) d_{q} s-\lim _{n \rightarrow \infty} t \int_{0}^{1} \frac{(1-q s)^{(\alpha-1)}}{\Gamma_{q}(\alpha)} g\left(s, x_{n}(s)\right) d_{q} s \\
& =\int_{0}^{t} \frac{(t-q s)^{(\alpha-1)}}{\Gamma_{q}(\alpha)} \lim _{n \rightarrow \infty} g\left(s, x_{n}(s)\right) d_{q} s-t \int_{0}^{1} \frac{(1-q s)^{(\alpha-1)}}{\Gamma_{q}(\alpha)} \lim _{n \rightarrow \infty} g\left(s, x_{n}(s)\right) d_{q} s
\end{aligned}
$$




$$
\begin{aligned}
& =\int_{0}^{t} \frac{(t-q s)^{(\alpha-1)}}{\Gamma_{q}(\alpha)} g(s, x(s)) d_{q} s-t \int_{0}^{1} \frac{(1-q s)^{(\alpha-1)}}{\Gamma_{q}(\alpha)} g(s, x(s)) d_{q} s \\
& =\mathcal{B} x(t)
\end{aligned}
$$

for all $t \in[0,1]$. This shows that $\mathcal{B}$ is continuous on $\bar{B}_{r}(0)$. It is enough to show that $\mathcal{B}\left(\bar{B}_{r}(0)\right)$ is a uniformly bounded and equicontinuous set in $X$. First we note that

$$
\begin{aligned}
|\mathcal{B} x(t)| & =\left|\int_{0}^{t} \frac{(t-q s)^{(\alpha-1)}}{\Gamma_{q}(\alpha)} g(s, x(s)) d_{q} s-t \int_{0}^{1} \frac{(1-q s)^{(\alpha-1)}}{\Gamma_{q}(\alpha)} g(s, x(s)) d_{q} s\right| \\
& \leq \int_{0}^{t} \frac{(t-q s)^{(\alpha-1)}}{\Gamma_{q}(\alpha)}|g(s, x(s))| d_{q} s+\int_{0}^{1} \frac{(1-q s)^{(\alpha-1)}}{\Gamma_{q}(\alpha)}|g(s, x(s))| d_{q} s \\
& \leq 2 \int_{0}^{1} \frac{(1-q s)^{(\alpha-1)}}{\Gamma_{q}(\alpha)} \gamma(s) \Omega(\|x\|) d_{q} s \\
& =\frac{2}{\Gamma_{q}(\alpha+1)}\|\gamma\| \Omega(r)
\end{aligned}
$$

for all $t \in[0,1]$. Taking supremum over the interval $[0,1]$ yields

$$
\|\mathcal{B} x\| \leq \frac{2}{\Gamma_{q}(\alpha+1)}\|\gamma\| \Omega(r)
$$

for all $x \in \bar{B}_{r}(0)$. This shows that $\mathcal{B}$ is uniformly bounded on $\bar{B}_{r}(0)$.

Next we show that $\mathcal{B}$ is an equicontinuous set in $X$. Let $t_{1}, t_{2} \in[0,1]$ with $t_{1}<t_{2}$. Then we have

$$
\begin{aligned}
& \left|\mathcal{B} x\left(t_{2}\right)-\mathcal{B} x\left(t_{1}\right)\right| \\
& \leq\left|\int_{0}^{t_{2}} \frac{\left(t_{2}-q s\right)^{(\alpha-1)}}{\Gamma_{q}(\alpha)} g(s, x(s)) d_{q} s-\int_{0}^{t_{1}} \frac{\left(t_{1}-q s\right)^{(\alpha-1)}}{\Gamma_{q}(\alpha)} g(s, x(s)) d_{q} s\right| \\
& \quad+\left(t_{2}-t_{1}\right) \int_{0}^{1} \frac{(1-q s)^{(\alpha-1)}}{\Gamma_{q}(\alpha)} g(s, x(s)) d_{q} s \\
& \leq\|\gamma\| \Omega(r) \int_{0}^{t_{1}}\left[\frac{\left(t_{2}-q s\right)^{(\alpha-1)}}{\Gamma_{q}(\alpha)}-\frac{\left(t_{1}-q s\right)^{(\alpha-1)}}{\Gamma_{q}(\alpha)}\right] d_{q} s \\
& \quad+\|\gamma\| \Omega(r) \int_{t_{1}}^{t_{2}} \frac{\left(t_{2}-q s\right)^{(\alpha-1)}}{\Gamma_{q}(\alpha)} d_{q} s+\|\gamma\| \Omega(r)\left(t_{2}-t_{1}\right) \int_{0}^{1} \frac{(1-q s)^{(\alpha-1)}}{\Gamma_{q}(\alpha)} d_{q} s .
\end{aligned}
$$

Obviously the right-hand side of the above inequality tends to zero independently of $x \in \bar{B}_{r}(0)$ as $t_{2}-t_{1} \rightarrow 0$. Therefore, it follows by the Arzelá-Ascoli theorem that $\mathcal{B}$ is a completely continuous operator on $\bar{B}_{r}(0)$.

Step 3. $M k<1$, that is, (c) of Lemma 3.2 holds.

This is obvious by $\left(\mathrm{H}_{3}\right)$ since we have $M=\left\|B\left(\bar{B}_{r}(0)\right)\right\|=\sup \left\{\|\mathcal{B} x\|: x \in \bar{B}_{r}(0)\right\}=$ $\frac{2}{\Gamma_{q}(\alpha+1)}\|\gamma\| \Omega(r)$ and $k=\|\phi\|$.

Thus the conditions (a), (b), and (c) of Lemma 3.2 are satisfied. Hence, either the conclusion (i) or the conclusion (ii) of Lemma 3.2 holds. We show that the conclusion (ii) is not possible. Let $x$ be a solution of the operator equation $x=\mathcal{A} x \mathcal{B} x$ with $\|x\|=r$ for some 
$\lambda, 0<\lambda<1$. Then we have

$$
\begin{aligned}
|x(t)|= & |\mathcal{A} x(t)||\mathcal{B} x(t)| \\
= & |f(t, x(t))|\left|\int_{0}^{t} \frac{(t-q s)^{(\alpha-1)}}{\Gamma_{q}(\alpha)} g(s, x(s)) d_{q} s-t \int_{0}^{1} \frac{(1-q s)^{(\alpha-1)}}{\Gamma_{q}(\alpha)} g(s, x(s)) d_{q} s\right| \\
\leq & {[|f(t, x(t))-f(t, 0)|+|f(t, 0)|] } \\
& \times\left|\int_{0}^{t} \frac{(t-q s)^{(\alpha-1)}}{\Gamma_{q}(\alpha)} g(s, x(s)) d_{q} s-t \int_{0}^{1} \frac{(1-q s)^{(\alpha-1)}}{\Gamma_{q}(\alpha)} g(s, x(s)) d_{q} s\right| \\
\leq & {\left[\phi(t)|x(t)|+F_{0}\right] 2 \Omega(\|x\|) \int_{0}^{1} \frac{(1-q s)^{(\alpha-1)}}{\Gamma_{q}(\alpha)} \gamma(s) d_{q} s } \\
\leq & {\left[\|\phi\|\|x\|+F_{0}\right] \frac{2}{\Gamma_{q}(\alpha+1)}\|\gamma\| \Omega(\|x\|) . }
\end{aligned}
$$

Thus

$$
r \leq \frac{\frac{2 F_{0}}{\Gamma_{q}(\alpha+1)}\|\gamma\| \Omega(r)}{1-\frac{2\|\phi\|}{\Gamma_{q}(\alpha+1)}\|\gamma\| \Omega(r)},
$$

which is a contradiction to (3.3), and hence the conclusion (ii) is not valid. Consequently, the conclusion (i) is valid, and hence the problem (1.1) has a solution on $\bar{B}_{r}(0)$. This completes the proof.

Example 3.4 Consider the boundary value problem

$$
\left\{\begin{array}{l}
D_{1 / 2}^{3 / 2}\left(\frac{x(t)}{\sin x+2}\right)=\frac{1}{4} \cos x(t), \quad 0<t<1 \\
x(0)=x(1)=0
\end{array}\right.
$$

Here $f(t, x)=\sin x+2, g(t, x)=\frac{1}{4} \cos x$. Observe that $\left(\mathrm{H}_{1}\right)$ and $\left(\mathrm{H}_{2}\right)$ hold with $\phi(t)=1$ and $\gamma(t)=\frac{1}{4}, \Omega(r)=1$, respectively. With the given data,

$$
\frac{2\|\phi\|}{\Gamma_{q}(\alpha+1)}\|\gamma\| \Omega(r)=\frac{1}{2 \Gamma_{q}(5 / 2)} \simeq 0.419959<1
$$

and we can choose $r$ such that $r>1.448031$. Hence the conclusion of Theorem 3.3 applies to the problem (3.7).

\section{Multi-valued case}

Let $L^{1}([0,1], \mathbb{R})$ be the Banach space of measurable functions $x:[0,1] \rightarrow \mathbb{R}$ which are Lebesgue integrable and normed by $\|x\|_{L^{1}}=\int_{0}^{1}|x(t)| d t$.

Now, we recall some basic concepts for multi-valued maps [30, 31].

For a normed space $(X,\|\cdot\|)$, let $\mathcal{P}_{c l}(X)=\{Y \in \mathcal{P}(X): Y$ is closed $\}, \mathcal{P}_{b}(X)=\{Y \in$ $\mathcal{P}(X): Y$ is bounded $\}, \mathcal{P}_{c p}(X)=\{Y \in \mathcal{P}(X): Y$ is compact $\}$, and $\mathcal{P}_{c p, c v}(X)=\{Y \in \mathcal{P}(X)$ : $Y$ is compact and convex\}. A multi-valued map $G: X \rightarrow \mathcal{P}(X)$ is convex (closed) valued if $G(x)$ is convex (closed) for all $x \in X$. The map $G$ is bounded on bounded sets if $G(\mathbb{B})=\bigcup_{x \in \mathbb{B}} G(x)$ is bounded in $X$ for all $\mathbb{B} \in \mathcal{P}_{b}(X)$ (i.e. $\left.\sup _{x \in \mathbb{B}}\{\sup \{|y|: y \in G(x)\}\}<\infty\right)$. $G$ is called upper semicontinuous (u.s.c.) on $X$ if for each $x_{0} \in X$, the set $G\left(x_{0}\right)$ is a 
nonempty closed subset of $X$, and if for each open set $N$ of $X$ containing $G\left(x_{0}\right)$, there exists an open neighborhood $\mathcal{N}_{0}$ of $x_{0}$ such that $G\left(\mathcal{N}_{0}\right) \subseteq N$. $G$ is said to be completely continuous if $G(\mathbb{B})$ is relatively compact for every $\mathbb{B} \in \mathcal{P}_{b}(X)$. If the multi-valued map $G$ is completely continuous with nonempty compact values, then $G$ is u.s.c. if and only if $G$ has a closed graph, i.e., $x_{n} \rightarrow x_{*}, y_{n} \rightarrow y_{*}, y_{n} \in G\left(x_{n}\right)$ imply $y_{*} \in G\left(x_{*}\right)$. $G$ has a fixed point if there is $x \in X$ such that $x \in G(x)$. The fixed point set of the multi-valued operator $G$ will be denoted by Fix $G$. A multi-valued map $G:[0 ; 1] \rightarrow \mathcal{P}_{c l}(\mathbb{R})$ is said to be measurable if for every $y \in \mathbb{R}$, the function

$$
t \mapsto d(y, G(t))=\inf \{|y-z|: z \in G(t)\}
$$

is measurable.

Definition 4.1 A multi-valued map $F:[0,1] \times \mathbb{R} \rightarrow \mathcal{P}(\mathbb{R})$ is said to be Carathéodory if

(i) $t \mapsto F(t, x)$ is measurable for each $x \in \mathbb{R}$;

(ii) $x \mapsto F(t, x)$ is upper semicontinuous for almost all $t \in[0,1]$.

Further a Carathéodory function $F$ is called $L^{1}$-Carathéodory if

(iii) there exists a function $g \in L^{1}\left([0,1], \mathbb{R}^{+}\right)$such that

$$
\|F(t, x)\|=\sup \{|v|: v \in F(t, x)\} \leq \mathrm{g}(t)
$$

for all $x \in \mathbb{R}$ and for a.e. $t \in[0,1]$.

For each $y \in C([0,1], \mathbb{R})$, define the set of selections of $F$ by

$$
S_{F, y}:=\left\{v \in L^{1}([0,1], \mathbb{R}): v(t) \in F(t, y(t)) \text { for a.e. } t \in[0,1]\right\} .
$$

The following lemma is used in the sequel.

Lemma 4.2 ([32]) Let $X$ be a Banach space. Let $F:[0,1] \times \mathbb{R} \rightarrow \mathcal{P}_{c p, c v}(X)$ be an $L^{1}$-Carathéodory multi-valued map and let $\Theta$ be a linear continuous mapping from $L^{1}([0,1], X)$ to $C([0, T], X)$. Then the operator

$$
\Theta \circ S_{F}: C([0,1], X) \rightarrow \mathcal{P}_{c p, c v}(C([0,1], X)), \quad x \mapsto\left(\Theta \circ S_{F}\right)(x)=\Theta\left(S_{F, x}\right)
$$

is a closed graph operator in $C([0,1], X) \times C([0,1], X)$.

Our second main result for the multi-valued problem (1.2) is based on the following fixed point theorem due to Dhage [28].

Lemma 4.3 Let $X$ be a Banach algebra and let $A: X \rightarrow X$ be a single-valued and $B: X \rightarrow$ $\mathcal{P}_{c p, c v}(X)$ be a multi-valued operator satisfying:

(a) A is a single-valued Lipschitz operator with a Lipschitz constant $k$,

(b) B is compact and upper semicontinuous,

(c) $2 M k<1$, where $M=\|B(X)\|$.

Then either

(i) the operator inclusion $x \in A \times B x$ has a solution, or

(ii) the set $\mathcal{E}=\{u \in X \mid \mu u \in A u B u, \mu>1\}$ is unbounded. 
Definition 4.4 A function $x \in A C^{1}([0,1], \mathbb{R})$ is called a solution of the problem (1.2) if there exists a function $v \in L^{1}([0,1], \mathbb{R})$ with $v(t) \in F(t, x(t))$, a.e. on $[0,1]$ such that $D_{q}^{\alpha}\left(\frac{x(t)}{f(t, x(t))}\right)=v(t)$, a.e. on $[0,1]$ and $x(0)=x(1)=0$.

Theorem 4.5 Assume that $\left(\mathrm{H}_{1}\right)$ holds. In addition we suppose that:

$\left(\mathrm{A}_{1}\right) \quad F:[0,1] \times \mathbb{R} \rightarrow \mathcal{P}(\mathbb{R})$ is $L^{1}$-Carathéodory and has nonempty compact and convex values;

$\left(\mathrm{A}_{2}\right)$ there exists a positive real number $\tilde{r}$ such that

$$
\begin{gathered}
\tilde{r}>\frac{\frac{2 F_{0}}{\Gamma_{q}(\alpha)}\|g\|_{L_{q}^{1}}}{1-\frac{2\|\phi\|}{\Gamma_{q}(\alpha)}\|g\|_{L_{q}^{1}}}, \\
\text { where } \frac{2\|g\|_{L_{q}^{1}}}{\Gamma_{q}(\alpha)}\|\phi\|<\frac{1}{2}, F_{0}=\sup _{t \in[0,1]}|f(t, 0)|,\|g\|_{L_{q}^{1}}=\int_{0}^{1}|g(t)| d_{q} t .
\end{gathered}
$$

Then the boundary value problem (1.2) has at least one solution on $[0,1]$.

Proof To transform the problem (1.2) into a fixed point problem, we define an operator $\mathcal{N}: X \rightarrow \mathcal{P}(X)$ as

$$
\begin{aligned}
\mathcal{B}(x)= & \{h \in X: \\
& \left.h(t)=f(t, x(t))\left(\int_{0}^{t} \frac{(t-q s)^{(\alpha-1)}}{\Gamma_{q}(\alpha)} v(s) d_{q} s-t \int_{0}^{1} \frac{(1-q s)^{(\alpha-1)}}{\Gamma_{q}(\alpha)} v(s) d_{q} s\right), v \in S_{F, x}\right\},
\end{aligned}
$$

where $X=C([0,1], \mathbb{R})$. Next we introduce two operators $\mathcal{A}_{1}: X \rightarrow X$ by

$$
\mathcal{A}_{1} x(t)=f(t, x(t)), \quad t \in[0,1],
$$

and $\mathcal{B}_{1}: X \rightarrow \mathcal{P}(X)$ by

$$
\begin{aligned}
\mathcal{B}_{1}(x)= & \{h \in X: \\
& \left.h(t)=\int_{0}^{t} \frac{(t-q s)^{(\alpha-1)}}{\Gamma_{q}(\alpha)} v(s) d_{q} s-t \int_{0}^{1} \frac{(1-q s)^{(\alpha-1)}}{\Gamma_{q}(\alpha)} v(s) d_{q} s, v \in S_{F, x}\right\} .
\end{aligned}
$$

Obviously $\mathcal{N}(x)=\mathcal{A}_{1} x \mathcal{B}_{1} x$. We shall show that the operators $\mathcal{A}_{1}$ and $\mathcal{B}_{1}$ satisfy all the conditions of Lemma 4.3. The proof is structured into a sequence of steps.

Step 1. We first show that $\mathcal{A}_{1}$ is Lipschitz on X, i.e., (a) of Lemma 4.3 holds.

The proof is similar to the one for the operator $\mathcal{A}$ in Step 1 of Theorem 3.3.

Step 2. Now we show that the multi-valued operator $\mathcal{B}_{1}$ is compact and upper semicontinuous on $X$, i.e. (b) of Lemma 4.3 holds.

Let us first show that $\mathcal{B}_{1}$ has convex values.

Let $u_{1}, u_{2} \in \mathcal{B}_{1} x$. Then there are $v_{1}, v_{2} \in S_{F, x}$ such that

$$
u_{i}(t)=\int_{0}^{t} \frac{(t-q s)^{(\alpha-1)}}{\Gamma_{q}(\alpha)} v_{i}(s) d_{q} s-t \int_{0}^{1} \frac{(1-q s)^{(\alpha-1)}}{\Gamma_{q}(\alpha)} v_{i}(s) d_{q} s, \quad t \in[0,1], i=1,2 .
$$


For any $\theta \in[0,1]$, we have

$$
\begin{aligned}
\theta u_{1}(t)+(1-\theta) u_{2}(t)= & \int_{0}^{t} \frac{(t-q s)^{(\alpha-1)}}{\Gamma_{q}(\alpha)}\left[\theta u_{1}(s)+(1-\theta) u_{2}(s)\right] d_{q} s \\
& -t \int_{0}^{1} \frac{(1-q s)^{(\alpha-1)}}{\Gamma_{q}(\alpha)}\left[\theta u_{1}(s)+(1-\theta) u_{2}(s)\right] d_{q} s \\
= & \int_{0}^{t} \frac{(t-q s)^{(\alpha-1)}}{\Gamma_{q}(\alpha)} \bar{v}(s) d_{q} s-t \int_{0}^{1} \frac{(1-q s)^{(\alpha-1)}}{\Gamma_{q}(\alpha)} \bar{v}(s) d_{q} s,
\end{aligned}
$$

where $\bar{v}(t)=\theta v_{1}(t)+(1-\theta) v_{2}(t) \in F(t, x(t))$ for all $t \in[0,1]$. Hence $\theta u_{1}(t)+(1-\theta) u_{2}(t) \in$ $\mathcal{B}_{1} x$ and consequently $\mathcal{B}_{1} x$ is convex for each $x \in X$. As a result $\mathcal{B}_{1}$ defines a multi-valued operator $\mathcal{B}_{1}: X \rightarrow \mathcal{P}_{c v}(X)$.

Next we show that $\mathcal{B}_{1}$ maps bounded sets into bounded sets in $X$. To do this, let $Q$ be a bounded set in $X$. Then there exists a real number $\sigma>0$ such that $\|x\| \leq \sigma, \forall x \in Q$.

Now for each $h \in \mathcal{B}_{1} x$, there exists a $v \in S_{F, x}$ such that

$$
h(t)=\int_{0}^{t} \frac{(t-q s)^{(\alpha-1)}}{\Gamma_{q}(\alpha)} v(s) d_{q} s-t \int_{0}^{1} \frac{(1-q s)^{(\alpha-1)}}{\Gamma_{q}(\alpha)} v(s) d_{q} s
$$

Then for each $t \in[0,1]$,

$$
\begin{aligned}
|h(t)| & =\left|\int_{0}^{t} \frac{(t-q s)^{(\alpha-1)}}{\Gamma_{q}(\alpha)} v(s) d_{q} s-t \int_{0}^{1} \frac{(1-q s)^{(\alpha-1)}}{\Gamma_{q}(\alpha)} v(s) d_{q} s\right| \\
& \leq \int_{0}^{t} \frac{(t-q s)^{(\alpha-1)}}{\Gamma_{q}(\alpha)} \mathrm{g}(s) d_{q} s+\int_{0}^{1} \frac{(1-q s)^{(\alpha-1)}}{\Gamma_{q}(\alpha)} \mathrm{g}(s) d_{q} s \\
& \leq 2 \int_{0}^{1} \frac{(1-q s)^{(\alpha-1)}}{\Gamma_{q}(\alpha)} \mathrm{g}(s) d_{q} s \\
& =\frac{2}{\Gamma_{q}(\alpha)}\|\mathrm{g}\|_{L_{q}^{1}} .
\end{aligned}
$$

This further implies that

$$
\|h\| \leq \frac{2}{\Gamma_{q}(\alpha)}\|\mathrm{g}\|_{L_{q}^{1}}
$$

and so $\mathcal{B}_{1}(X)$ is uniformly bounded.

Next we show that $\mathcal{B}_{1}$ maps bounded sets into equicontinuous sets. Let $Q$ be, as above, a bounded set and $h \in \mathcal{B}_{1} x$ for some $x \in Q$. Then there exists a $v \in S_{F, x}$ such that

$$
h(t)=\int_{0}^{t} \frac{(t-q s)^{(\alpha-1)}}{\Gamma_{q}(\alpha)} v(s) d_{q} s-t \int_{0}^{1} \frac{(1-q s)^{(\alpha-1)}}{\Gamma_{q}(\alpha)} v(s) d_{q} s
$$

Then for any $t_{1}, t_{2} \in[0,1]$ with $t_{1} \leq t_{2}$ we have

$$
\begin{aligned}
\left|h\left(t_{2}\right)-h\left(t_{1}\right)\right| \leq & \left|\int_{0}^{t_{2}} \frac{\left(t_{2}-q s\right)^{(\alpha-1)}}{\Gamma_{q}(\alpha)} v(s) d_{q} s-\int_{0}^{t_{1}} \frac{\left(t_{1}-q s\right)^{(\alpha-1)}}{\Gamma_{q}(\alpha)} v(s) d_{q} s\right| \\
& +\left(t_{2}-t_{1}\right) \int_{0}^{1} \frac{(1-q s)^{(\alpha-1)}}{\Gamma_{q}(\alpha)} v(s) d_{q} s
\end{aligned}
$$




$$
\begin{aligned}
\leq & \int_{0}^{t_{1}}\left[\frac{\left(t_{2}-q s\right)^{(\alpha-1)}}{\Gamma_{q}(\alpha)}-\frac{\left(t_{1}-q s\right)^{(\alpha-1)}}{\Gamma_{q}(\alpha)}\right] \mathrm{g}(s) d_{q} s \\
& +\int_{t_{1}}^{t_{2}} \frac{\left(t_{2}-q s\right)^{(\alpha-1)}}{\Gamma_{q}(\alpha)} \mathrm{g}(s) d_{q} s \\
& +\left(t_{2}-t_{1}\right) \int_{0}^{1} \frac{(1-q s)^{(\alpha-1)}}{\Gamma_{q}(\alpha)} \mathrm{g}(s) d_{q} s,
\end{aligned}
$$

which tends to zero independently of $x \in Q$ as $t_{2}-t_{1} \rightarrow 0$.

Therefore it follows by the Arzelá-Ascoli theorem that $\mathcal{B}_{1}: C([0,1], \mathbb{R}) \rightarrow \mathcal{P}(C([0,1], \mathbb{R}))$ is completely continuous.

In our next step, we show that $\mathcal{B}_{1}$ has a closed graph. Let $x_{n} \rightarrow x_{*}, h_{n} \in \mathcal{B}_{1}\left(x_{n}\right)$ and $h_{n} \rightarrow h_{*}$. Then we need to show that $h_{*} \in \mathcal{B}_{1}$. Associated with $h_{n} \in \mathcal{B}_{1}\left(x_{n}\right)$, there exists $v_{n} \in S_{F, x_{n}}$ such that, for each $t \in[0,1]$,

$$
h_{n}(t)=\int_{0}^{t} \frac{(t-q s)^{(\alpha-1)}}{\Gamma_{q}(\alpha)} v_{n}(s) d_{q} s-t \int_{0}^{1} \frac{(1-q s)^{(\alpha-1)}}{\Gamma_{q}(\alpha)} v_{n}(s) d_{q} s .
$$

Thus it suffices to show that there exists $v_{*} \in S_{F, x_{*}}$ such that, for each $t \in[0,1]$,

$$
h_{*}(t)=\int_{0}^{t} \frac{(t-q s)^{(\alpha-1)}}{\Gamma_{q}(\alpha)} v_{*}(s) d_{q} s-t \int_{0}^{1} \frac{(1-q s)^{(\alpha-1)}}{\Gamma_{q}(\alpha)} v_{*}(s) d_{q} s .
$$

Let us consider the linear operator $\Theta: L^{1}([0,1], \mathbb{R}) \rightarrow C([0,1], \mathbb{R})$ given by

$$
f \mapsto \Theta(v)(t)=\int_{0}^{t} \frac{(t-q s)^{(\alpha-1)}}{\Gamma_{q}(\alpha)} v(s) d_{q} s-t \int_{0}^{1} \frac{(1-q s)^{(\alpha-1)}}{\Gamma_{q}(\alpha)} v(s) d_{q} s .
$$

Notice that

$$
\begin{aligned}
\left\|h_{n}(t)-h_{*}(t)\right\|= & \| \int_{0}^{t} \frac{(t-q s)^{(\alpha-1)}}{\Gamma_{q}(\alpha)}\left(v_{n}(s)-v_{*}(s)\right) d_{q} s \\
& -t \int_{0}^{1} \frac{(1-q s)^{(\alpha-1)}}{\Gamma_{q}(\alpha)}\left(v_{n}(s)-v_{*}(s)\right) d_{q} s \| \rightarrow 0, \quad \text { as } n \rightarrow \infty .
\end{aligned}
$$

Thus, it follows by Lemma 4.2 that $\Theta \circ S_{F}$ is a closed graph operator. Further, we have $h_{n}(t) \in \Theta\left(S_{F, x_{n}}\right)$. Since $x_{n} \rightarrow x_{*}$, therefore, we have

$$
h_{*}(t)=\int_{0}^{t} \frac{(t-q s)^{(\alpha-1)}}{\Gamma_{q}(\alpha)} v_{*}(s) d_{q} s-t \int_{0}^{1} \frac{(1-q s)^{(\alpha-1)}}{\Gamma_{q}(\alpha)} v_{*}(s) d_{q} s
$$

for some $v_{*} \in S_{F, x_{*}}$.

As a result we find that the operator $\mathcal{B}_{1}$ is a compact and upper semicontinuous operator on $X$.

Step 3. Now we show that $2 M k<1$, i.e. (c) of Lemma 4.3 holds.

It is obvious in view of $\left(\mathrm{H}_{3}\right)$ with $M=\|B(X)\|=\sup \{|\mathcal{B} x|: x \in X\} \leq \frac{2}{\Gamma_{q}(\alpha)}\|\mathrm{g}\|_{L_{q}^{1}}$ and $k=\|\phi\|$.

Thus all the conditions of Lemma 4.3 are satisfied and, in consequence, it follows that either the conclusion (i) or the conclusion (ii) holds. We show that the conclusion (ii) is not possible. 
Let $u \in \mathcal{E}$ be arbitrary. Then we have $\lambda u(t) \in \mathcal{A}_{1} u(t) \mathcal{B}_{1} u(t)$ for $\lambda>1$ and there exists $v \in S_{F, x}$ such that, for any $\lambda>1$,

$$
u(t)=\lambda^{-1}[f(t, u(t))]\left(\int_{0}^{t} \frac{(t-q s)^{(\alpha-1)}}{\Gamma_{q}(\alpha)} v(s) d_{q} s-t \int_{0}^{1} \frac{(1-q s)^{(\alpha-1)}}{\Gamma_{q}(\alpha)} v(s) d_{q} s\right)
$$

for all $t \in[0,1]$. Thus we have

$$
\begin{aligned}
|u(t)| \leq & \lambda^{-1}|f(t, u(t))|\left(\int_{0}^{t} \frac{(t-q s)^{(\alpha-1)}}{\Gamma_{q}(\alpha)}|v(s)| d_{q} s+\int_{0}^{1} \frac{(1-q s)^{(\alpha-1)}}{\Gamma_{q}(\alpha)}|v(s)| d_{q} s\right) \\
\leq & {[|f(t, u(t))-f(t, 0)|+|f(t, 0)|] } \\
& \times\left(\int_{0}^{t} \frac{(t-q s)^{(\alpha-1)}}{\Gamma_{q}(\alpha)}|v(s)| d_{q} s+\int_{0}^{1} \frac{(1-q s)^{(\alpha-1)}}{\Gamma_{q}(\alpha)}|v(s)| d_{q} s\right) \\
\leq & 2\left[\|\phi\|\|u\|+F_{0}\right] \int_{0}^{1} \frac{(1-q s)^{(\alpha-1)}}{\Gamma_{q}(\alpha)} \mathrm{g}(s) d_{q} s \\
\leq & {\left[\|\phi\|\|u\|+F_{0}\right] \frac{2}{\Gamma_{q}(\alpha)}\|\mathrm{g}\|_{L_{q}^{1}}, }
\end{aligned}
$$

where $F_{0}=\sup _{t \in[0,1]}|f(t, 0)|$.

Consequently, with $\|u\|=\tilde{r}$, we have

$$
\tilde{r} \leq \frac{\frac{2 F_{0}}{\Gamma_{q}(\alpha)}\|\mathrm{g}\|_{L_{q}^{1}}}{1-\frac{2\|\phi\|}{\Gamma_{q}(\alpha)}\|\mathrm{g}\|_{L_{q}^{1}}} .
$$

Thus the condition (ii) of Theorem 4.3 does not hold in view of the condition (4.1). Therefore, the operator inclusion $x \in \mathcal{A}_{1} x \mathcal{B}_{1} x$ has a solution, and, in turn, the problem (1.2) has a solution on $[0,1]$. This completes the proof.

Example 4.6 Consider the problem

$$
\left\{\begin{array}{l}
D_{1 / 2}^{3 / 2}\left[\frac{x(t)}{t \sin x / 6+2}\right] \in F(t, x(t)), \quad 0<t<1 \\
x(0)=x(1)=0
\end{array}\right.
$$

where $F:[0,1] \times \mathbb{R} \rightarrow \mathcal{P}(\mathbb{R})$ is a multi-valued map given by

$$
t \rightarrow F(t, x)=\left[\frac{|x|^{3}}{10\left(|x|^{3}+3\right)}, \frac{|\sin x|}{9(|\sin x|+1)}+\frac{8}{9}\right] .
$$

For $f \in F$ we have

$$
|f| \leq \max \left(\frac{|x|^{3}}{10\left(|x|^{3}+3\right)}, \frac{|\sin x|}{9(|\sin x|+1)}+\frac{8}{9}\right) \leq 1, \quad x \in \mathbb{R} .
$$

Thus

$$
\|F(t, x)\|=\sup \{|y|: y \in F(t, x)\} \leq 1=\mathrm{g}(t), \quad x \in \mathbb{R} .
$$


Further, $\|\phi\|=1 / 6, \Gamma_{\frac{1}{2}}(3 / 2) \simeq 0.920875, \tilde{r}>12 /\left[3 \frac{1}{2}(3 / 2)-1\right]$ and

$$
\frac{2\|\phi\|\|\mathrm{g}\|_{L_{q}^{1}}}{\Gamma_{q}(\alpha)}=\frac{1}{3 \Gamma_{\frac{1}{2}}(3 / 2)} \simeq 0.361975<\frac{1}{2} .
$$

Thus all the conditions of Theorem 4.5 are satisfied and consequently, the problem (4.4) has a solution on $[0,1]$.

\section{Competing interests}

The authors declare that they have no competing interests.

\section{Authors' contributions}

Each of the authors, BA and SKN, contributed to each part of this work equally and read and approved the final version of the manuscript.

\section{Author details}

1 Department of Mathematics, Faculty of Science, King Abdulaziz University, P.O. Box 80203, Jeddah, 21589, Saudi Arabia.

${ }^{2}$ Department of Mathematics, University of loannina, loannina, 451 10, Greece.

\section{Authors' information}

Sotiris K Ntouyas is a member of Nonlinear Analysis and Applied Mathematics (NAAM) - Research Group at King Abdulaziz University, Jeddah, Saudi Arabia.

\section{Acknowledgements}

This paper was funded by Deanship of Scientific Research (DSR), King Abdulaziz University, Jeddah, Saudi Arabia. Therefore, the authors acknowledge with thanks DSR for the financial support.

Received: 6 May 2014 Accepted: 13 June 2014 Published: 23 Jul 2014

\section{References}

1. Podlubny, I: Fractional Differential Equations. Academic Press, San Diego (1999)

2. Kilbas, AA, Srivastava, HM, Trujillo, JJ: Theory and Applications of Fractional Differential Equations. North-Holland Mathematics Studies, vol. 204. Elsevier, Amsterdam (2006)

3. Sabatier, J, Agrawal, OP, Machado, JAT (eds.): Advances in Fractional Calculus: Theoretical Developments and Applications in Physics and Engineering. Springer, Dordrecht (2007)

4. Lakshmikantham, V, Leela, S, Vasundhara Devi, J: Theory of Fractional Dynamic Systems. Cambridge Scientific Publishers, Cambridge (2009)

5. Mainardi, F: Fractional Calculus and Waves in Linear Viscoelasticity: An Introduction to Mathematical Models. Imperial College Press, London (2010)

6. Diethelm, K: The Analysis of Fractional Differential Equations: An Application-Oriented Exposition Using Differential Operators of Caputo Type. Lecture Notes in Mathematics, vol. 2004. Springer, Berlin (2010)

7. Klafter, J, Lim, SC, Metzler, R (eds.): Fractional Dynamics in Physics. World Scientific, Singapore (2011)

8. Baleanu, D, Diethelm, K, Scalas, E, Trujillo, JJ: Fractional Calculus Models and Numerical Methods. Series on Complexity, Nonlinearity and Chaos. World Scientific, Boston (2012)

9. Ferreira, RAC: Nontrivial solutions for fractional $q$-difference boundary value problems. Electron. J. Qual. Theory Differ. Equ. 2010, 70 (2010)

10. Goodrich, CS: Existence and uniqueness of solutions to a fractional difference equation with nonlocal conditions. Comput. Math. Appl. 61, 191-202 (2011)

11. Atici, FM, Eloe, PW: Two-point boundary value problems for finite fractional difference equations. J. Differ. Equ. Appl. 17, 445-456 (2011)

12. Abdeljawad, T, Baleanu, D: Caputo $q$-fractional initial value problems and a $q$-analogue Mittag-Leffler function. Commun. Nonlinear Sci. Numer. Simul. 16, 4682-4688 (2011)

13. Ma, J, Yang, J: Existence of solutions for multi-point boundary value problem of fractional q-difference equation. Electron. J. Qual. Theory Differ. Equ. 2011, 92 (2011)

14. Graef, JR, Kong, L: Positive solutions for a class of higher order boundary value problems with fractional $q$-derivatives. Appl. Math. Comput. 218, 9682-9689 (2012)

15. Ahmad, B, Ntouyas, SK, Purnaras, IK: Existence results for nonlocal boundary value problems of nonlinear fractional q-difference equations. Adv. Differ. Equ. 2012, 140 (2012)

16. Ferreira, RAC: Positive solutions for a class of boundary value problems with fractional $q$-differences. Comput. Math. Appl. 61, 367-373 (2011)

17. Wu, GC, Baleanu, D: New applications of the variational iteration method - from differential equations to $q$-fractional difference equations. Adv. Differ. Equ. 2013, 21 (2013)

18. Alsaedi, A, Ahmad, B, Al-Hutami, H: A study of nonlinear fractional q-difference equations with nonlocal integral boundary conditions. Abstr. Appl. Anal. 2013, Article ID 410505 (2013)

19. Ahmad, B, Nieto, JJ, Alsaedi, A, Al-Hutami, H: Existence of solutions for nonlinear fractional q-difference integral equations with two fractional orders and nonlocal four-point boundary conditions. J. Franklin Inst. 351, 2890-2909 (2014) 
20. Al-Salam, WA: Some fractional q-integrals and q-derivatives. Proc. Edinb. Math. Soc. 15, 135-140 (1966-1967)

21. Agarwal, RP: Certain fractional q-integrals and q-derivatives. Proc. Camb. Philos. Soc. 66, 365-370 (1969)

22. Annaby, MH, Mansour, ZS: q-Fractional Calculus and Equations. Lecture Notes in Mathematics, vol. 2056. Springer, Berlin (2012)

23. Ammi, M, El Kinani, E, Torres, D: Existence and uniqueness of solutions to functional integro-differential fractional equations. Electron. J. Differ. Equ. 2012, 103 (2012)

24. El Borai, M, Abbas, M: On some integro-differential equations of fractional orders involving Carathéodory nonlinearities. Int. J. Mod. Math. 2, 41-52 (2007)

25. Zhao, Y, Sun, S, Han, Z, Li, Q: Theory of fractional hybrid differential equations. Comput. Math. Appl. 62, 1312-1324 (2011)

26. Sun, S, Zhao, Y, Han, Z, Li, Y: The existence of solutions for boundary value problem of fractional hybrid differential equations. Commun. Nonlinear Sci. Numer. Simul. 17, 4961-4967 (2012)

27. Dhage, BC: Nonlinear functional boundary value problems involving Carathéodory. Kyungpook Math. J. 46, 427-441 (2006)

28. Dhage, BC: Existence results for neutral functional differential inclusions in Banach algebras. Nonlinear Anal. 64 1290-1306 (2006)

29. Rajkovic, PM, Marinkovic, SD, Stankovic, MS: On q-analogues of Caputo derivative and Mittag-Leffler function. Fract. Calc. Appl. Anal. 10, 359-373 (2007)

30. Hu, S, Papageorgiou, N: Handbook of Multivalued Analysis, Volume I: Theory. Kluwer Academic, Dordrecht (1997)

31. Deimling, K: Multivalued Differential Equations. de Gruyter, Berlin (1992)

32. Lasota, A, Opial, Z: An application of the Kakutani-Ky Fan theorem in the theory of ordinary differential equations. Bull. Acad. Pol. Sci., Sér. Sci. Math. Astron. Phys. 13, 781-786 (1965)

10.1186/1687-1847-2014-199

Cite this article as: Ahmad and Ntouyas: Fractional $q$-difference hybrid equations and inclusions with Dirichlet

boundary conditions. Advances in Difference Equations 2014, 2014:199

\section{Submit your manuscript to a SpringerOpen ${ }^{\ominus}$ journal and benefit from:}

- Convenient online submission

Rigorous peer review

- Immediate publication on acceptance

- Open access: articles freely available online

- High visibility within the field

- Retaining the copyright to your article 